\title{
Modified wake oscillator model for vortex-induced motion prediction of low aspect ratio structures
}

Mohd Asamudin A Rahman a , Wan Norazam Wan Hussin a, Mohd Hairil

Mohd $^{\mathrm{a}}$, Fatimah Noor Harun ${ }^{\mathrm{b}}$, Lee Kee Quen ${ }^{\mathrm{c}}$, Jeom Kee Paik ${ }^{\mathrm{d}, \mathrm{e}, \mathrm{f}}$

${ }^{a}$ School of Ocean Engineering, Universiti Malaysia Terengganu, Terengganu, Malaysia

${ }^{b}$ School of Informatics and Applied Mathematics, Universiti Malaysia Terengganu, Terengganu, Malaysia

${ }^{c}$ Malaysia-Japan International Institute of Technology, Universiti Teknologi Malaysia, Kuala Lumpur, Malaysia

${ }^{d}$ Department Mechanical Engineering, University College London, London, UK

${ }^{e}$ Department of Naval Architecture and Ocean Engineering, Pusan National University, Busan, Korea

${ }^{f}$ The Korea Ship and Offshore Research Institute (Lloyd's Register Foundation Research Centre of Excellence), Busan, Korea

Corresponding author: Mohd Asamudin A Rahman.

Email address: mohdasamudin@umt.edu.my 


\title{
Modified wake oscillator model for vortex-induced motion prediction of low aspect ratio structures
}

\begin{abstract}
Floating structures, such as Spar platform, are likely to experience significant motions while operation due to Vortex-induced Motion (VIM). The main features of VIM include aspect ratio and mass ratio of the structure. This paper focuses on further development of a previously published semi-empirical method called wake oscillator model (WOM) for VIM, which aims to derive a mathematical correlation in modelling all features of the phenomenon. Analytical solution is proposed to coupled WOM and structure oscillator model based on the Van der Pol equation by integrating mass ratio, $\mathrm{m}^{*}=4.36$, and various low aspect ratios between 0.3 and 2.0. Additionally, a new approach was initiated to embed several empirical parameters linked to the aspect ratio in the existing WOM. It was found the aspect ratio parameter in the modified WOM significantly affected the performance of the model for estimation of VIM on floating structures.
\end{abstract}

Keywords: wake oscillator model; vortex-induced motions; low aspect ratio; Van der Pol equation

Subject classification codes: include these here if the journal requires them

\subsection{Introduction}

Vortex-Induced Vibration (VIV) appears to be a significant consideration in designing moorings, risers, and offshore platforms. Recently, topics pertaining to VIV phenomena have been extensively reviewed, wherein a substantial number of successful approaches in VIV prediction have been adopted (see Khalak and Williamson 1999; Govardhan and Williamson 2000; Sarpkaya 2004; Stappenbelt and O' Neill 2007; Srinil and Zanganeh 2012; Gonçalves et al. 2013). As for structures built offshore, a number of factors need to be weighed in that may significantly affect costs of operation and maintenance. Various types of offshore structures may experience VIV and Vortex-induced Motion (VIM). Hence, this study focused on a circular cylindrical structure with low aspect 
ratio, particularly the SPAR platform, to arrive at a formula for VIM estimation. Analyses on the structural responses of this particular structure are indeed crucial, where structural reliability and serviceability are among the significant factors that need to be taken into account for analyses purpose. This has been portrayed in several recent investigations concerning VIM phenomenon on offshore structures (see Rahman and Thiagarajan 2015; Rahman et al. 2016; Cagney and Balabani 2016; Hirabayashi 2016; Thorsen et al. 2016; Gonçalves et al. 2018).

A variety of approaches have been applied to the solution of VIV in the attempt of devising a viable method from experimental, numerical, and analytical stances. Nonetheless, the analytical approach seems to be far from perfect. Additionally, researches on semi-empirical model for VIM phenomenon have yet to receive as much attention as VIV does. VIM is another way of distinguishing the VIV phenomenon on both infinite and finite (floating) structures. The primary features of the floating structure are low aspect ratio and the ratio of mass and fluid displaced mass is equal to one $\left(m^{*}=1\right)$. Therefore, this research formulated the VIM response of the floating structure since the fluid flow employed an analytical modelling called wake oscillator model (WOM).

The WOM refers to a self-exciting and self-limiting solution that has been used to describe both rigid and elastic cylinders. This model usually considers 2-dimensional (2D) flow, apart from generating the equations of cylinder oscillators and fluid oscillator by independent means, which are later applied to predict the response of coupled fluid structure systems. Gao et al. (2019), Kang et al. (2018), Kurushina et al., (2018), Xu et al. (2017), Farshidianfar and Zanganeh (2010), Jin and Dong (2016), Low and Srinil (2016), Postnikov et al. (2017), Violette et al. (2007), Hussin et al. (2017), Xu et al. (2015), and Facchinetti et al. (2004) are among the contributors to the vast 
research work in the area of WOM.

To the best of the authors' knowledge, no study has assessed the prediction of low aspect ratio and low mass ratio for VIM using WOM based on the characteristics of circular cylindrical floating structures. With that, the main focus of this study is the modification to a previous WOM in predicting VIM of low aspect ratio structures. Of particular interest, this study is aimed at estimating both motion amplitude and vibration/shedding frequencies of the structure with varying low aspect ratios. The present work dealing with a modified model which was based on previously published prediction models. Derivation of the newly modified WOM was conducted and a new formulation to include the effect of aspect ratio in the prediction model was introduced, tested and validated with empirical outcomes and experimental results from the literature.

\subsection{Mathematical modelling}

A circular cylindrical structure was considered in this study to illustrate a semiempirical model, as illustrated in Figure 1. The analytical models were based on a 1 degree of freedom (DOF) of a rigid circular cylinder of diameter, D, which was elastically mounted. The bluff body surface experienced fluctuating pressure, varied with time as the body was subjected to fluid flow with uniform velocity, U. Additionally, the vortices were alternately shed and separated from the bluff body, wherein oscillating pressure fields were developed. If the body is free to move in the transverse direction of the fluid flow, the resultant periodic net forces are bound to interact with the structural movement, originating from the vortex shedding induced vibration. The parameters applied in the model are tabulated in Table 1. 


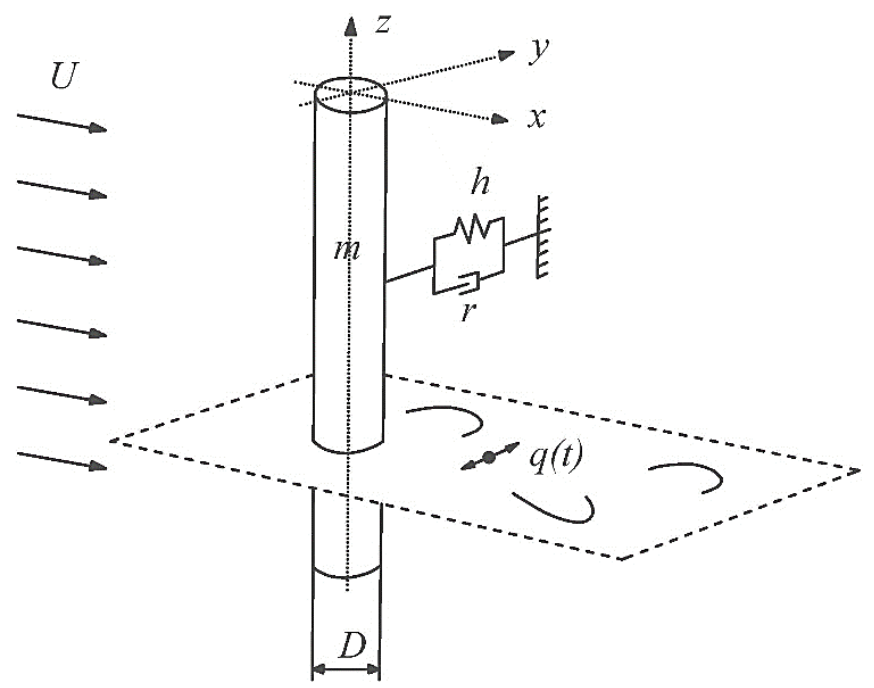

Figure 1. Illustration model of coupled structure and wake oscillator for 2D vortexinduced vibration (Facchinetti et al., 2004; Postnikov et al., 2017).

Table 1. Parameter values.

\begin{tabular}{ll}
\hline Parameter & Value \\
\hline Strouhal Number, $S_{t}$ & 0.2 \\
Van Der Pol Parameter, $\varepsilon$ & 0.3 \\
Scaling of the coupling force, A & 12 \\
Damping Coefficient, $\gamma$ & 0.8 \\
Drag Coefficient, $C_{D}$ & 2.0 \\
\hline
\end{tabular}

\subsection{Structural oscillator}

The in-plane form displacement $\mathrm{Y}$ of the cylinder, and the crosswise to fluid flow can be stated in a linear oscillator, as defined by Facchinetti et al. (2004).

$$
m \ddot{Y}+r \dot{Y}+h Y=S
$$

where overdots $(\cdot)$ refer to derivatives with respect to dimensional time, $\mathrm{T}$. The inviscid inertia effects of fluid (Blevins 1990), which denotes the mass, $m$, are composed of two parts: $m_{s}$ represents the mass of the structure, while $m_{f}$ stands for fluid-added mass. The stiffness coefficient in Equation (1), $h$, is associated to external effects (Facchinetti et al. 2004). In developing the 2D model, all parameters, including 
mass, damping, and stiffness, had been defined per unit length. The fluid hydrodynamic effects on the structure consisted of two parts: the basic fluid effects, $m_{f}$, and $r_{f}$ that directly influences the structure, while the effects of vortices modelled as a forcing term had been as defined by Facchinetti et al. (2004) and given in the following:

$$
S=\frac{1}{2} \rho U^{2} D C_{L}
$$

where $C_{L}$ refers to fluctuating lift coefficient. The definition of structural angular frequency, $\Omega_{s}=\sqrt{h / m}$, structure reduced damping, $\xi=r_{s} / 2 m \Omega_{s}$, and vortexshedding frequency, $\Omega_{\mathrm{f}}=2 \pi \mathrm{S}_{\mathrm{t}} \mathrm{U} / \mathrm{D}$ had been explained by Facchinetti et al. (2004), hence constructing Equation (1) as follows:

$$
\ddot{Y}+\left(2 \xi \Omega_{s}+\frac{\gamma}{\mu} \Omega_{f}\right) \dot{Y}+\Omega_{f}^{2} Y=\frac{S}{M}
$$

\subsection{Wake oscillator}

Equation of wake in Equation (1) was applied to couple with the equation of wake structure in Equation (4). Nature fluctuations of the vortex street was modelled by a nonlinear oscillator in order to comply with the van der Pol equation (Nayfeh 1993).

$$
\ddot{q}+\varepsilon \Omega_{f}\left(q^{2}-1\right) \dot{q}+\Omega_{f}^{2} q=F
$$

The dimensionless wake variable associated with the fluctuating lift coefficient had been considered as the main variable, which can be defined as $q=2 C_{L} / C_{L O}$, where $C_{L}$ stands for instantaneous lift coefficient, while $C_{L 0}$ represents reference lift coefficient of a stationary cylinder under vortex shedding. The right hand side forcing term of Equation (4), F, models the effect of cylinder motion on the wake dynamics. 


\subsection{Coupling of wake and structural oscillators}

In order to normalise the method, the dimensionless time $t=\Omega_{s} T$ and space coordinate $y=Y / D$ were initiated by Facchinetti et al. (2004). Equations (3) and (4) lead to the coupled fluid-structure dynamical system.

$$
\ddot{y}+\left(2 \xi \delta+\frac{\gamma}{\mu}\right) \dot{y}+\delta^{2} y=s, \quad \ddot{q}+\varepsilon\left(q^{2}-1\right) \dot{q}+q=f
$$

where $\delta$ refers to the reduced angular frequency of the structure, which is also related to reducing flow velocity (Facchinetti et al. 2004).

$$
\delta=\frac{1}{\mathrm{~s}_{\mathrm{t}} \mathrm{U}_{\mathrm{r}}}, \quad U_{r}=2 \pi U /\left(\Omega_{\mathrm{s}} D\right)
$$

and $s=S /\left(D \Omega_{f}^{2} m\right)$, considering the definition of $\mathrm{q}$, $\mathrm{S}$ becomes as given below:

$$
s=M q, \quad M=\frac{\frac{C_{L 0}}{2}}{8 \pi^{2} S_{t} \mu}
$$

A number of researchers have been using various coupling models, such as displacement coupling $f=A y$, velocity coupling $f=A \dot{y}$, and acceleration coupling $f=A \ddot{y}$, to define this forcing term. As such, this study focuses on the use of acceleration coupling mainly because acceleration coupling have successfully analysed to model all features of VIV, such as lock-in domain, hysteresis, and Griffin plot (Facchinetti et al. 2004). Therefore, Equation (5) appears as given in the following;

$$
\begin{gathered}
\ddot{y}+\left(2 \xi \delta+\frac{\gamma}{\mu}\right) \dot{y}+\delta^{2} y=M q \\
\ddot{q}+\varepsilon\left(q^{2}-1\right) \dot{q}+q=A \ddot{y}
\end{gathered}
$$

A and $\varepsilon$ refer to the parameters that can be derived from empirical data on the wake dynamics. Typically, $A=12$ and $\varepsilon=0.3$ (Facchinetti et al. 2004). The overdots $(\cdot)$ of 
Equations (8) and (9) are derivatives with respect to dimensionless time, $t$.

\subsection{Coupling system}

Here, the coupled fluid structure system had been considered for Equation (5) as replacement in finding a solution in the form described by Facchinetti et al. (2004). It reads as follows:

$$
y=y_{0} \cos (\omega t), \quad q=q_{0} \cos (\omega t-\varphi)
$$

Substitution of Equation (10) into Equation (5) to retrieve both amplitude and phase of the linear transfer function between structure displacement and fluid variable is given in the following:

$$
\frac{y_{0}}{q_{0}}=\frac{M}{\sqrt{\left(\delta^{2}-\omega^{2}\right)^{2}+\left(2 \xi \delta+\frac{\gamma}{\mu}\right)^{2} \omega^{2}}}, \tan \varphi=\frac{-\left(2 \xi \delta+\frac{\gamma}{\mu}\right) \omega}{\delta^{2}-\omega^{2}}
$$

Substituting after considering the only main harmonic contribution of the nonlinearities in the wake oscillator, Equation (5) led to equations on $q_{0}$ and $\omega$ that refer to amplitude and angular frequency, respectively (Facchinetti et al. 2004).

$$
\begin{aligned}
& q_{0}=2 \sqrt{1+\frac{A M}{\varepsilon} \frac{\left(2 \xi \delta+\frac{\gamma}{\mu}\right) \omega^{2}}{\left(\delta^{2}-\omega^{2}\right)^{2}+\left(2 \xi \delta+\frac{\gamma}{\mu}\right)^{2} \omega^{2}}} \\
& \omega^{6}-\left[1+2 \delta^{2}-(2 \xi \delta+\gamma / \mu)^{2}\right] \omega^{4}-\left[-2 \delta^{2}+(2 \xi \delta+\gamma / \mu)^{2}-\delta^{4}\right] \omega^{2}-\delta^{4}+ \\
& A M\left(\omega^{2}-\delta^{2}\right) \omega^{2}=0
\end{aligned}
$$

The angular frequency $\omega$, derived from Equation (13), produced one or three 
positive real roots. Based on the solutions, angular frequency using coupling Equations (11) and (12) raised the amplitude $y_{0}$ of the structure motions. The present coupled model was validated and discussed earlier by Hussin et al. (2017) for 2D prediction of VIV.

\subsection{Coupling model validation}

The validation were made succeeded previous coupling model by Hussin et al. (2017) and comparison between analytical model by Facchinetti et al. (2004) and experimental data from Govardhan and Williamson (2000) are shown in Figure 2. For comparison sake, the low mass ratio $\mathrm{m}^{\wedge *}=0.52$ and low reduced damping $\xi=0.0052$ are used. Based on the validation acquired, it can be seen that there was a good agreement between the present coupled model and the existing analytical model by Facchinetti et al. (2004). However, the model in could not predict higher response of the structure due to limitation on the parameters involved. 


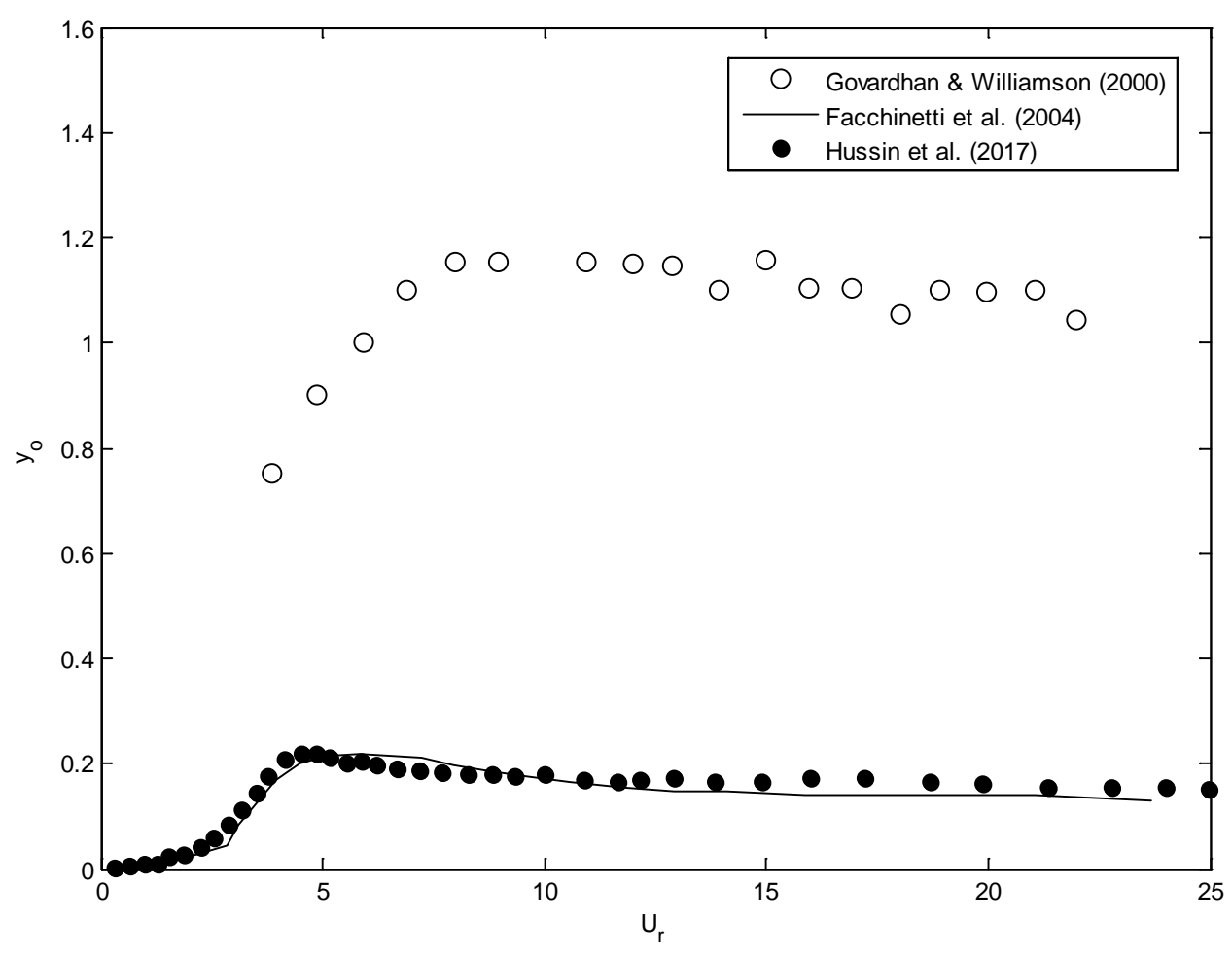

Figure 2. Oscillation amplitude response at low mass ratio $m *=0.52$ and low reduced damping $\xi=0.0052$ (Hussin et al. 2017).

The validation outcome was retrieved from solving Equations (11), (12) and (13), based on the coupling model introduced previously by Hussin et al. (2017). A significant parameter that affected the VIV refers to low mass ratio (Sarpkaya 2004). In a structure with low mass ratio, the cylinder oscillates at a higher amplitude when compared to a structure with high mass ratio. Fig. 3 illustrates the structural oscillation amplitude $y_{0}$ as a function of the reduced velocity $U_{r}$ at the value of mass ratio $m^{*}=2.36$ and 3.68. The result was compared with experimental work performed by Stappenbelt and O' Neill (2007) for low mass ratio structures. 


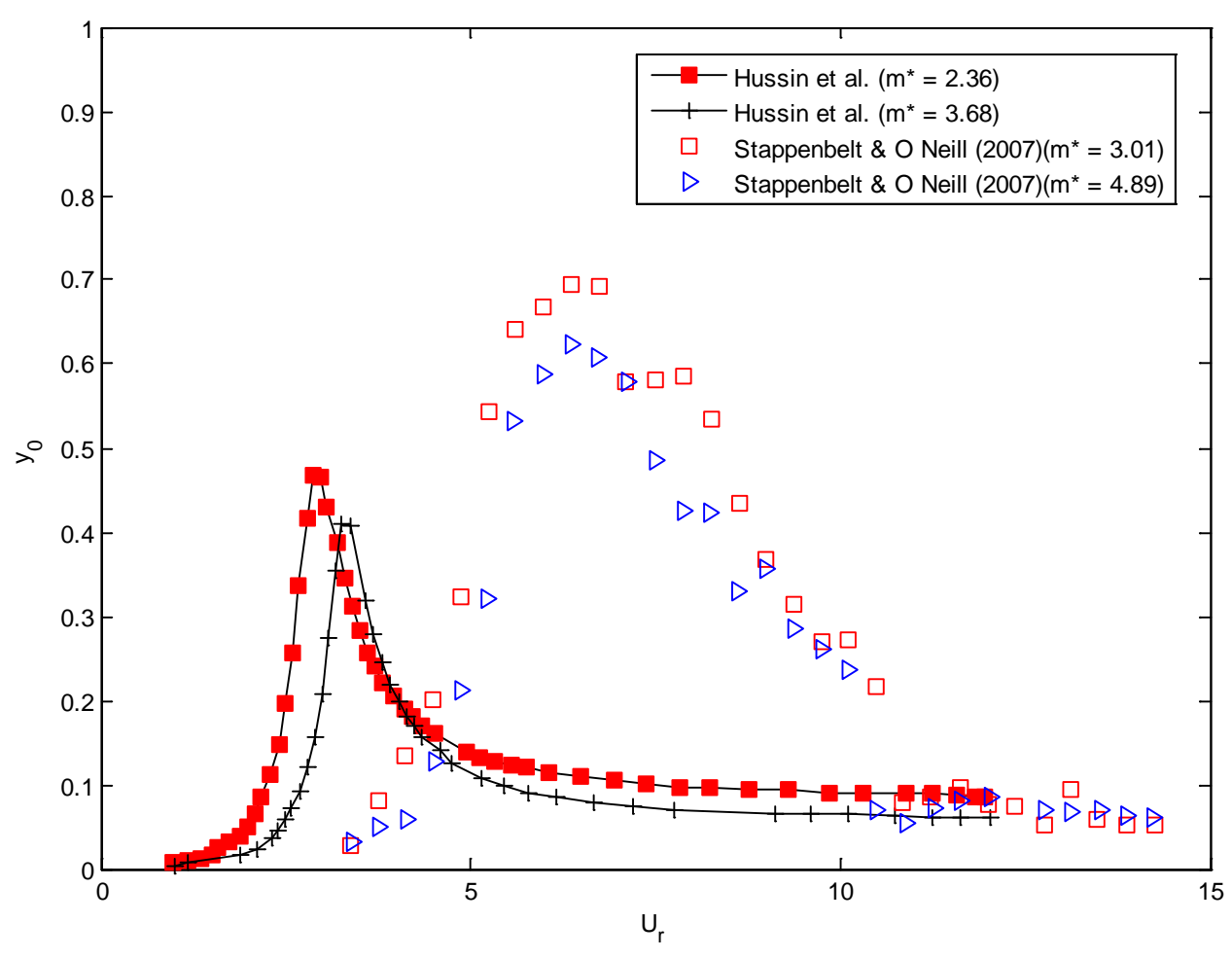

Figure 3. Oscillation amplitude response at different mass ratios.

It can be seen that amplitude motions escalated with decrease in mass ratio. In fact, the figure illustrates similar trends for all mass ratios in terms of maximum amplitude, but contrasting outcomes for onset and range of lock-in. Typically, the onset of lock-in shifts to a higher level reduced the velocities for higher mass ratio. The investigation of vortex shedding at each mass ratio strengthened earlier observations concerning the amplitude response region. It was revealed that when the fluid condition seemed more congested as the cylinder vibrated in the water, varied changes in natural frequency were noted. By increasing the natural frequency, it was discovered that increment in the reduction of velocity enhanced decrease in mass along the span of lock-in (Stappenbelt and O' Neill (2007). Nevertheless, the lock-in range did not differ much across all mass ratios. This was probably due to the limitation of WOM in 
capturing various synchronisation of the frequency over differing mass ratios. Nevertheless, the previous published model exhibited its capability in predicting the oscillation amplitudes for the desired mass ratios.

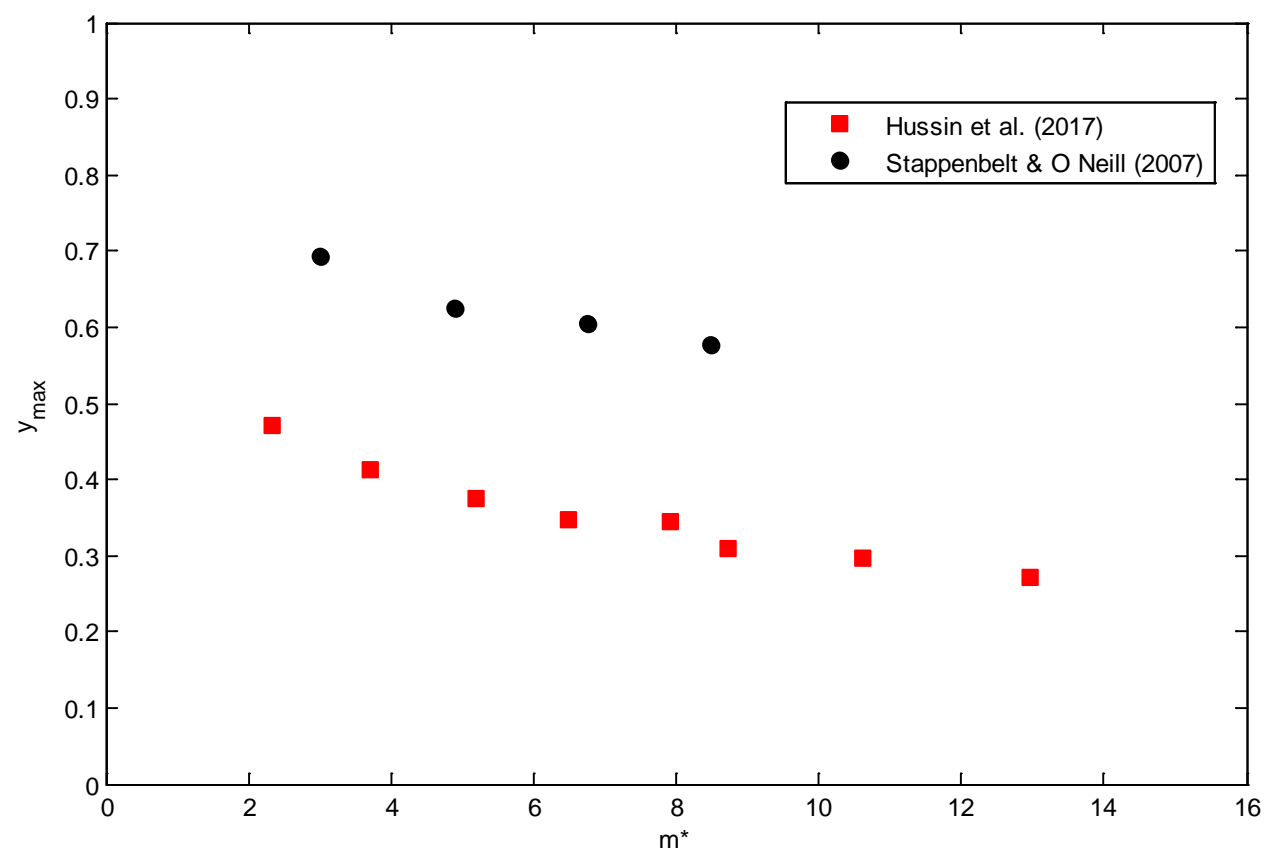

Figure 4. Amplitude maxima response plot versus different mass ratio.

The maximum amplitude response for various mass ratios displayed, generally, a decreasing trend with mass ratio (Fig. 4) (Sumer and Fredsoe 2006). Additionally, the higher mass ratio contributed to slight influence of the hydrodynamic mass on the vibration response of the structure. Changes in hydrodynamics mass affected the synchronisation of the shedding and the vibration frequencies, whereas widespread lock-in regions normally occur at a low mass ratio (Stappenbelt and O' Neill 2007; Sumer \& Fredsoe 2006). The present coupled model offers consistent trend evidences, especially when compared to those presented in the literature. However, the model only captures the trend qualitatively and hence a modification to the model is needed to 
quantitatively capture the experimental values from literature. The proposed modification to the model was based on the influence of aspect ratio on the development of WOM prediction model.

\subsection{Modification of coupling model using classical model}

The modified model was composed of van der Pol and fifth-order aerodynamic damping term initiated by Landl (1975), given as follows:

$$
\ddot{C}_{L}+\left(\dot{\alpha}-\dot{\beta} C_{L}^{2}+\dot{\lambda} C_{L}^{4}\right) \dot{C}_{L}+\Omega_{r} C_{L}=b \dot{y}
$$

where $\alpha, \dot{\beta}, \hat{\lambda}$, and $b$ are constants selected freely about the given problem, while $\Omega_{r}$ stands for minimised frequency of the structure. As a sample in the modified model, the damping term, $\varepsilon$, had been applied. Since the comparison between modified wake oscillator and van der Pol was required, it was assumed that $\alpha=\varepsilon, \dot{\beta} / \varepsilon=\beta$, and $\hat{\lambda} / \varepsilon=$ $\lambda$ (Farshidianfar and Dolatabadi 2013). Hence, Equation (14) turned into Equation (15) by extracting the cofactor, $\varepsilon$, and considering a general form of force in terms of $f$ by inserting $q / q_{o}=c_{L} / c_{L o}$ into it. It reads as follows:

$$
\ddot{q}+\varepsilon\left(1-\beta q^{2}+\lambda q^{4}\right) \dot{q}+\Omega_{r} q=f
$$

The Landl model was modified, while neglecting $\Omega_{r}$ so as to keep the recently modified model attributes intact, in comparison to the main classic model in Equation (5). Next, the final modified equation was introduced as suggested by Farshidianfar and Dolatabadi (2013), as given in the following:

$$
\ddot{q}+\varepsilon\left(1-\beta q^{2}+\lambda q^{4}\right) \dot{q}+q=f
$$


In accordance to Facchinetti et al. (2004) and Farshidianfar and Dolatabadi (2013), the present model was studied based on a higher-order model by replacing each displacement, velocity, and acceleration coupling terms as in Equation (9) in terms of $f$. By applying the straightforward method described by Farshidianfar and Dolatabadi (2013), the finalised results were retrieved so as to define a set of curves with limited cycle behaviour in a fourth-order equation, as follows;

$$
\begin{aligned}
& q_{0}, 1=\sqrt{\frac{\frac{2 \beta}{\lambda}+\left(\left(\frac{2 \beta}{\lambda}\right)^{2}-\frac{32}{\lambda}\right)^{0.5}}{2}} \\
& q_{0}, 2=\sqrt{\frac{\frac{2 \beta}{\lambda}-\left(\left(\frac{2 \beta}{\lambda}\right)^{2}-\frac{32}{\lambda}\right)^{0.5}}{2}}
\end{aligned}
$$

A significant modification of the structural should be made if the equivalent $\lambda$ is selected for bifurcation point and $q_{0}$ is employed with positive square root sign. Coefficients $\beta$ and $\lambda$, which maximised $q_{0}$ using the value obtained from Equations (17) and (18) resulted in coefficient $\beta=0.0625$ and $\lambda=0.0005$, which indicated the exact values for modification. The classic WOM with variable coefficient $\beta$ had been weighed in. Thus, the dynamical equation built by Facchinetti et al. (2004) was retained, apart from maintaining amplitude $q_{0}$, where $\beta$ had an integral role, as follows:

$$
q_{0}=2 / \sqrt{\beta} \sqrt{1+\frac{A M}{\varepsilon} \frac{\left(2 \xi \delta+\frac{\gamma}{\mu}\right) \omega^{2}}{\left(\delta^{2}-\omega^{2}\right)^{2}+\left(2 \xi \delta+\frac{\gamma}{\mu}\right)^{2} \omega^{2}}}
$$

In order to extend the capability of the formulation to predict VIM, the parameter of aspect ratio $(L / D)$ was considered. The dimensionless parameter of aspect 
ratio, $(L / D)$, was initiated because it was believed that the parameter can strongly influence VIM prediction via fundamental of VIV phenomenon. Hence, based on Equation (14), some modifications were made to the independently chosen parameters, where $\alpha, \dot{\beta}$, and $\lambda$ were defined as follows:

$$
\dot{\alpha}=\varepsilon, \quad \frac{\dot{\beta}}{\varepsilon}=\beta \xi /\left(\frac{L}{D}\right) \text { and } \dot{\lambda} / \varepsilon=\lambda \xi /\left(\frac{L}{D}\right)
$$

where the variable that reduced damping, $\xi$, in still water (\%) was employed as a comparison modified model and another parameter, also coupling equation, remained unchanged. By substituting Equation (20) into Equation (14) and following the rest of the steps, Equation (19) can be expressed as follows:

$$
q_{0}=\frac{2}{\sqrt{\beta \xi /(L / D)}} \sqrt{1+\frac{A M}{\varepsilon} \frac{\left(2 \xi \delta+\frac{\gamma}{\mu}\right) \omega^{2}}{\left(\delta^{2}-\omega^{2}\right)^{2}+\left(2 \xi \delta+\frac{\gamma}{\mu}\right)^{2} \omega^{2}}}
$$

Introduction of this coefficient results in the following modification of the system of equations of motion. This refers to the new modified coupling model with impacts of the structural aspect ratio $(L / D)$ on hydrodynamic forces and responses so as to gain a better view of the VIM phenomenon prediction via classical VIV problem.

\subsection{Results and discussion}

\subsection{Calibration and validation on the selected experimental data}

Fig. 5 portrays the response amplitude of the modified model in comparison with the literature for mass ratio, $m^{*}=4.36$ and $m^{*}=1.0$ with $L / D=2.0$. The normalized 
amplitude, $y_{0}$ for the modified WOM was compared with the analytical model by Facchinetti et al. (2004) and experiments by Stappenbelt and O’Neill (2007), Khalak and Williamson (1999), Triantafyllou et al. (2003), and Morse et al. (2008). As expected, the agreement with the literature is acceptable for initial and upper branch of the vibration. This was mainly due to different trends was observed in experiments with low aspect ratio as reported by Goncalves et al. (2013). The data used for comparative purposes in this figure, most of them, was collected with a higher aspect ratio than the present work on low aspect ratio, at least higher than $\mathrm{L} / \mathrm{D}=13$. As for the comparison with Facchinetti et al. (2004), it was noted that in regime $2<U_{r}<6$, both the models adhered to the pattern of empirical data from the literature. As for $U_{r}>4$, the existing model started to shift away from the experimental values, which contradicts with the present modified model of Equation (21). This yield showcases that the modified model had successfully captured the phenomenon appeared to be in good agreement with the empirical data reported by Gonçalves et al. (2013). The present modified model had successfully predicted the VIM phenomenon for respective mass and aspect ratio, thus further emphasising the significance of $L / D$ in the modified model. This could be attributed to the $3 \mathrm{D}$ effects from $L / D$ parameters, in comparison to the existing $2 \mathrm{D}$ model. It was discovered that the modified model could be applied to capture the pattern and the behaviour of empirical values, especially when compared to the existing model. 


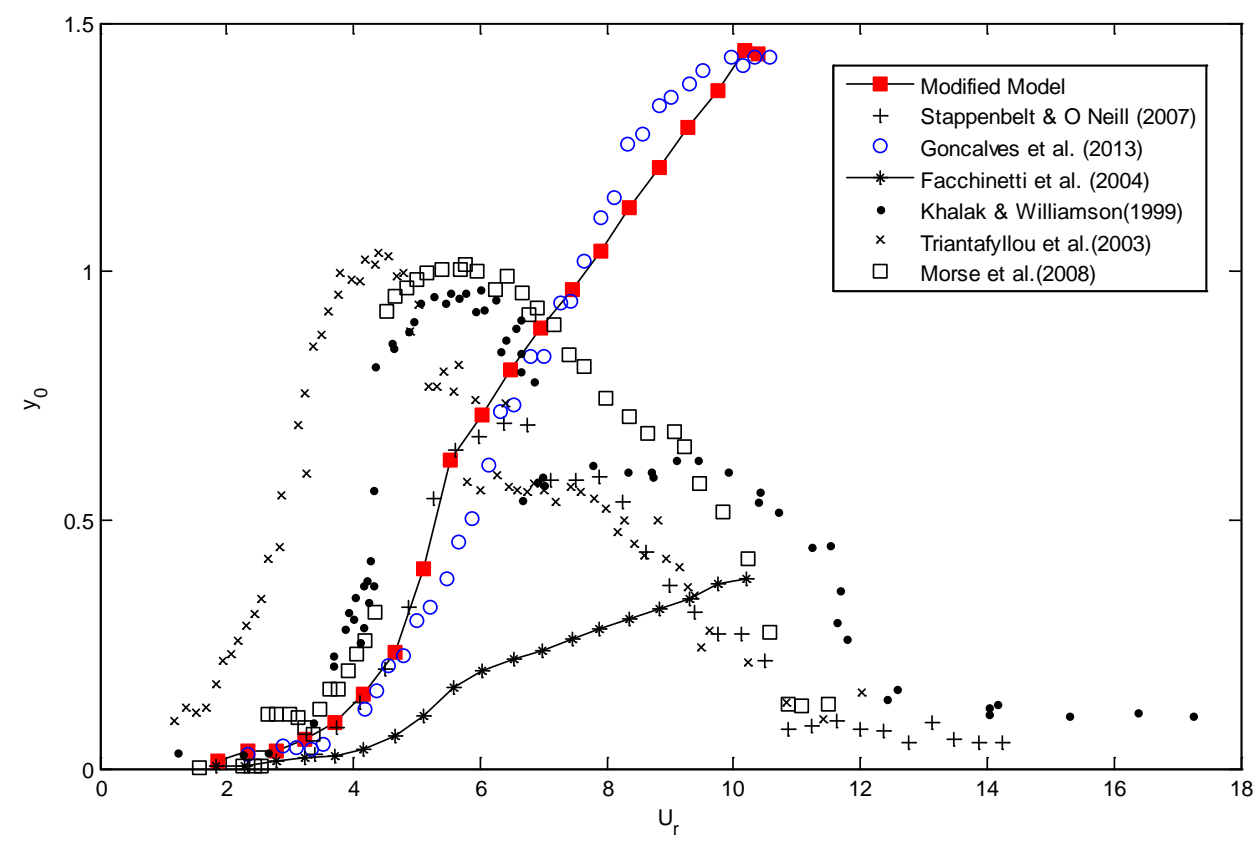

Figure 5. Comparison of amplitudes response of modified WOM with analytical model by Facchinetti et al. (2004) and experimental data, $\mathrm{y}_{0}$ as a function reduced velocity, $\mathrm{U}_{\mathrm{r}}$ for circular cylinders.

\subsection{Influence of $L / D$ on $m *=4.36$}

The outcomes for various $L / D$ were obtained by solving Equations (11), (13), and (21) of the modified WOM. Comparison was made with empirical yields obtained by Gonçalves et al. (2013), who investigated the influence of $L / D$ upon low aspect ratio circular cylinders. Fig. 6 illustrates the transverse amplitudes for the mass ratio of $m^{*}=4.36$ for the following aspect ratios of $L / D=0.30,0.40,0.50,0.75,1.00$, 1.50, and 2.00. The modified model captured most of the experimental trends from high to low $L / D$, where the amplitudes reduced with smaller aspect ratio. The findings show that it was indeed possible to correlate the end effects in the analytical model for VIM prediction. Reduction in the maximum amplitude with increased mass ratio had been due to the 3D effects of the shorter submerged cylinder length. This significantly minimised the lift coefficient and the drag force on the structure (Rahman et al., 2015). 

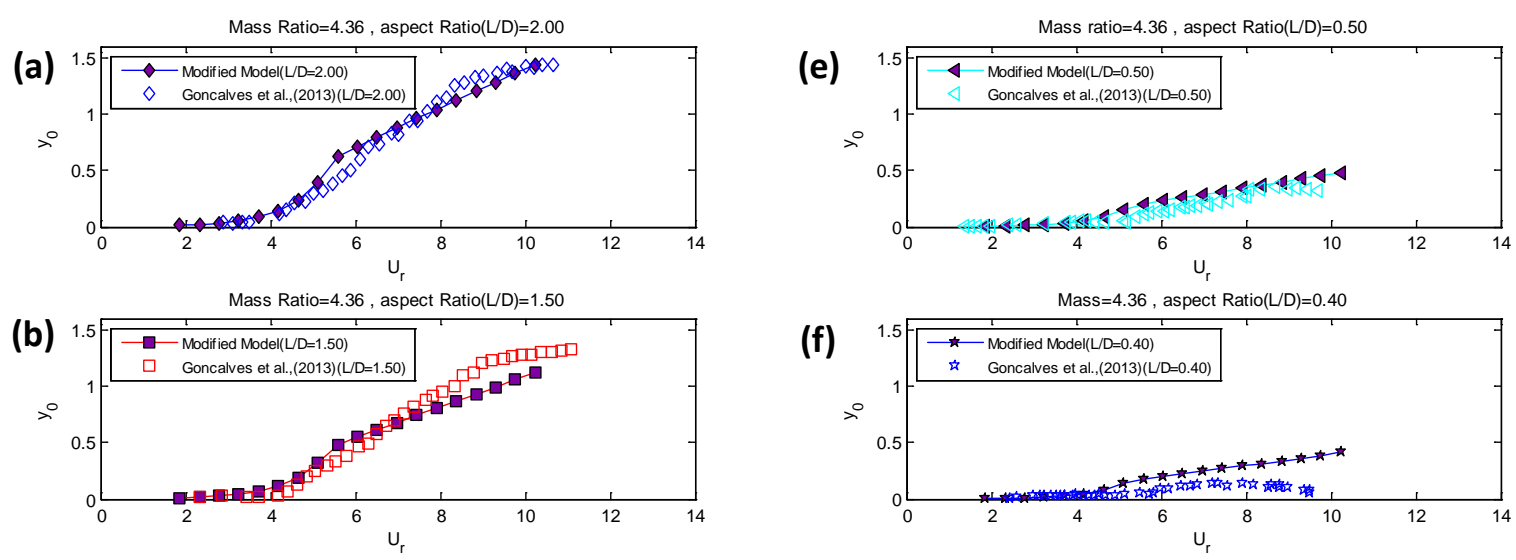

(f)
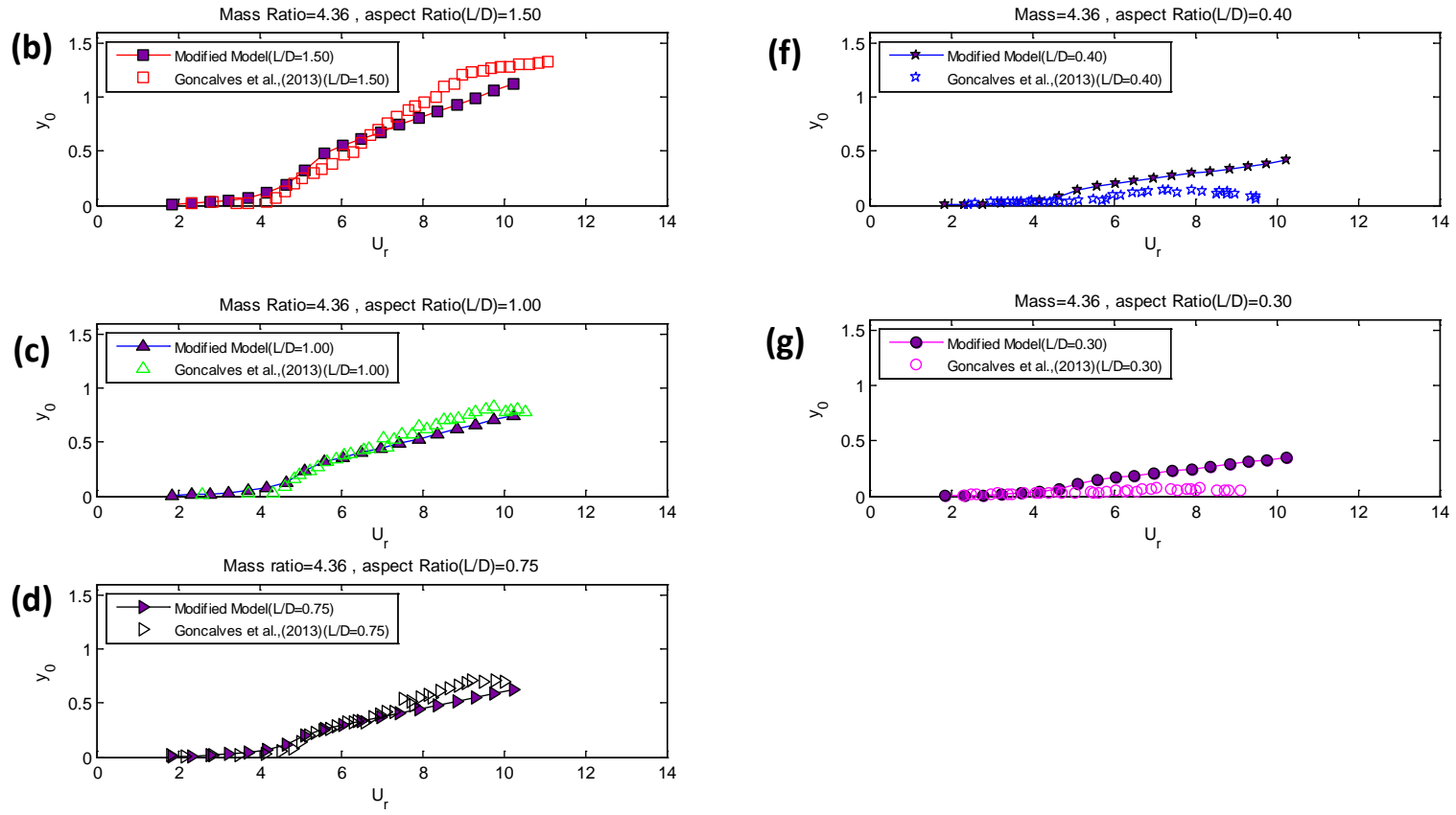

Figure 6. Amplitudes response of modified WOM, $\mathrm{y}_{0}$ as a function reduced velocity, $\mathrm{U}_{\mathrm{r}}$ for circular cylinders with mass ratio, $\mathrm{m}^{*}=4.36$ for all aspect ratio, $\mathrm{L} / \mathrm{D}$ (a)2.00, (b)1.50, (c)1.00, (d)0.75, (e)0.50, (f)0.40, and (g) 0.30 .

It is worth noting that the modified model failed in closely capturing the motion amplitude for the two lowest aspect ratios, $\mathrm{L} / \mathrm{D}=0.4$ and 0.3 . The predicted values were slightly higher, in comparison to empirical outcomes. Alternating vortices were not observed as the correlation length of the vortex formation was demolished by the turbulent flow due to the end condition and free surface (Gonçalves et al. 2013). As for these two aspect ratios, synchronisation was absent for the shedding and the structure frequency in the experiment. Nevertheless, a limitation was noted in the modified model, where the model failed to capture intricate end conditions as the aspect ratio (L/D) went below 0.4 . 
Fig. 7 presents the frequency response of modified WOM, in comparison to empirical data. In order to compare the dimensionless frequency, the frequency $f^{*}=\omega / \delta$ was considered in this study (Facchinetti et al. 2004). It was noted that the modified model displayed coupled mode flutter (CMF) region. Hence, the model was able to describe both vibrating and vortex shedding frequency of the lock-in region. Besides, the modified WOM successfully predicted the value of frequency ratio from the experimental work.

(a)

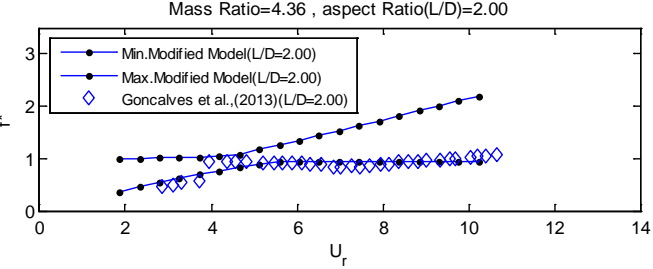

(b)

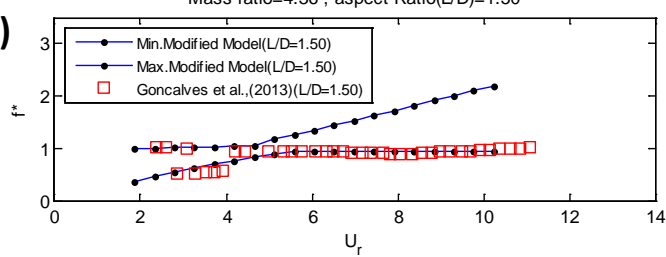

(c)

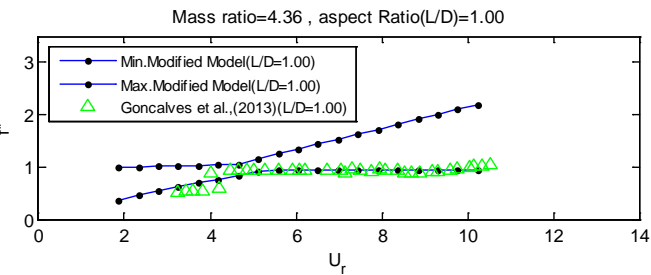

(d)

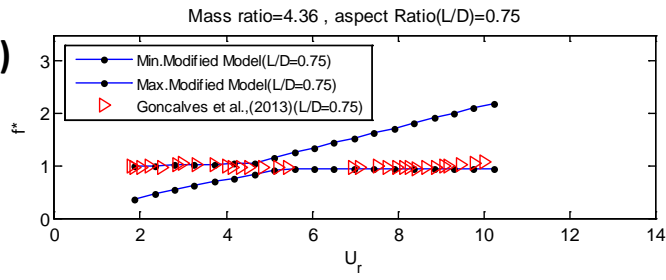

(e)

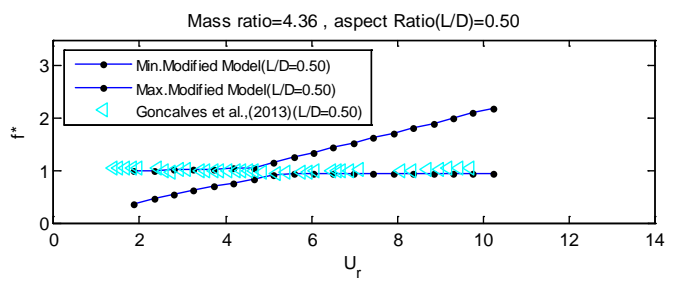

(f)

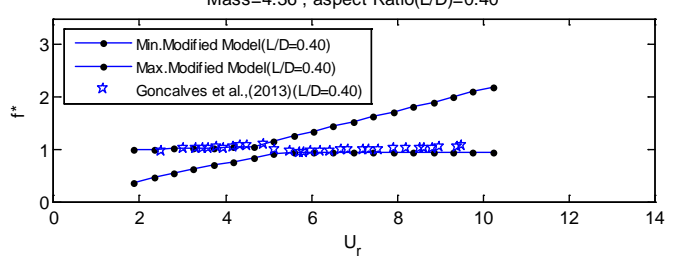

(g)

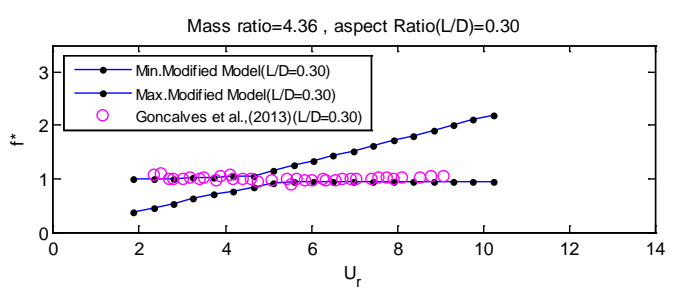

Figure 7. Frequency ratio of modified WOM, $f^{*}$ as a function of reduced velocity, $U_{r}$ for circular cylinders with mass ratio, $\mathrm{m}^{*}=4.36$ and aspect ratio, $\mathrm{L} / \mathrm{D}$ (a)2.00, (b)1.50, (c)1.00, (d)0.75, (e)0.50, (f)0.40, and (g) 0.30 (Gonçalves et al. 2013). 


\subsection{Conclusion}

A modified WOM is presented in this study to model VIM, wherein the main focus was to investigate the impact of aspect ratio on the performance of prediction model. Modified WOM incorporated the aspect ratio parameter, L/D, into the wake equation that subsequently solved with the coupled Van der pol equation. The outcomes of the modified wake oscillator were calibrated and validated with free vibration measurements over a range of aspect ratios. The model indeed possessed the capability to describe the most important trends in the free vibration measurements of low aspect ratio where most of vibration amplitude values were captured. The lock-in range of the free vibration was predicted acceptably over a range of aspect ratios. For aspect ratios $(\mathrm{L} / \mathrm{D})<0.4$, the model predicted a slightly higher amplitude of cylinder vibration with increment in flow velocity, when compared to empirical data. Notably, the modified wake oscillator displayed its ability in modelling free vibration of 3D structure for VIM prediction. The model predictions can probably be enhanced by improving the coupling terms of Equation (21), where the correction factor could be integrated into the aspect ratio parameter with thorough validation and calibration of experimental data. Surprisingly, this study had successfully predicted VIM of floaters by initiating a parameter of the aspect ratio ranging from 0.2 to 2.0 to capture the 3D phenomenon.

\section{Acknowledgement}

This work was supported by the Ministry of Higher Education Malaysia under Fundamental Research Grant Scheme FRGS/1/15/59416. 


\section{References}

1. Blevins R. 1990. Flow-induced vibration. Van Nostrand Reinhold, New York.

2. Cagney N. \& Balabani S. 2016. Fluid forces acting on a cylinder undergoing streamwise vortex-induced vibrations. Journal of Fluids and Structures. 62:147155.

3. Facchinetti ML, De Langre E \& Biolley F. 2004. Coupling of structure and wake oscillators in vortex-induced vibrations, Journal of Fluids and Structures. 19: $123-140$.

4. Farshidianfar A \& Dolatabadi N. 2013. Modified higher-order wake oscillator model for vortex-induced vibration of circular cylinders. Acta Mechanica. 224(7): 1441-1456.

5. Farshidianfar A, \& Zanganeh H. 2010. A modified wake oscillator model for vortex-induced vibration of circular cylinders for a wide range of mass-damping ratio. Journal of Fluids and Structures, 26(3): 430-441.

6. Gao Y, Zou L, Zong Z, Takagi S, Kang Y. 2019. Numerical prediction of vortex-induced vibrations of a long flexible cylinder in uniform and linear shear flows using a wake oscillator model, Ocean Engineering. 171: 157-171.

7. Gonçalves RT, Meneghini JR, and Fujarra ALC. 2018. Vortex-induced vibration of floating circular cylinders with very low aspect ratio. Ocean Engineering. 154: 234-251.

8. Gonçalves RT, Rosetti GF, Franzini GR, Meneghini JR \& Fujarraa ALC. 2013. Two-degree-of-freedom vortex-induced vibration of circular cylinders with very low aspect ratio and small mass ratio. Journal of Fluids and Structures. 39: 237257.

9. Govardhan, R., \& Williamson, C. (2000). Modes of vortex formation and frequency response of a freely vibrating cylinder. Journal of Fluid Mechanics, 420, 85-130. doi:10.1017/S0022112000001233

10. Hirabayashi S. 2016. Numerical analysis of vortex-induced motion of twodimensional circular cylinder by lattice Boltzmann method. Journal of Marine Science and Technology. 21(3): 426-433.

11. Hussin WNW, Harun FN, Mohd MH and Rahman MAA. 2017. Analytical modelling prediction by using wake oscillator model for vortex-induced vibrations, Journal of Mechanical Engineering and Sciences. 11(4): 3116-3128. 
12. Jin Y, \& Dong P. 2016. A novel Wake Oscillator Model for simulation of crossflow vortex induced vibrations of a circular cylinder close to a plane boundary. Ocean Engineering. 117:57-62.

13. Kang Z, Zhang C \& Chang R. 2018. A higher-order nonlinear oscillator model for coupled cross-flow and in-line VIV of a circular cylinder. Ships and Offshore Structures. 13(5): 488-503.

14. Khalak A \& Williamson CHK. 1999. Motions, forces and mode transitions in vortex-induced vibrations at low mass-damping. Journal of Fluids and Structures. 13(7-8): 813-851.

15. Kurushina V, Pavlovskaia E, Postnikov A, Wiercigroch M. 2018. Calibration and comparison of VIV wake oscillator models for low mass ratio structures, International Journal of Mechanical Sciences. 142-143: 547-560.

16. Landl R. 1975. A mathematical model for vortex-excited vibrations of bluff bodies. Journal of Sound and Vibration. 42(2):219-234.

17. Low YM \& Srinil N. 2016. VIV fatigue reliability analysis of marine risers with uncertainties in the wake oscillator model. Engineering Structures. 106:96-108.

18. Morse T.L., Govardhan R.N, and Williamson C.H.K (2008). "The effects of end conditions on the vortex-induced vibration of cylinders,” Journal of Fluids and Structures, Vol 24, pp 1227-1239.

19. Nayfeh AH. 1993. Introduction to Perturbation Techniques. Wiley, New York.

20. Postnikov A, Pavlovskaia E \& Wiercigroch M. 2017. 2DOF CFD calibrated wake oscillator model to investigate vortex-induced vibrations. International Journal of Mechanical Sciences. 127:176-190.

21. Rahman MAA, Leggoe J, Thiagarajan KP, Mohd MH \& Paik JK .2016. Numerical simulations of vortex-induced vibrations on vertical cylindrical structure with different aspect ratios. Ships and Offshore Structures. 11(4): 405423.

22. Rahman MAA \& Thiagarajan KP. 2015. Experiments on Vortex-induced Vibration of a Vertical Cylindrical Structure: Effect of Low Aspect Ratio. International Journal of Automotive and Mechanical Engineering. 11(1): 25152530.

23. Sarpkaya, T. 2004. A critical review of the intrinsic nature of vortex induced vibrations. J Fluid Structure. 19: 389-447. 
24. Srinil N \& Zanganeh H. 2012. Modelling of coupled cross-flow/in-line vortexinduced vibrations using double Duffing and van der Pol oscillators. Ocean Engineering. 53:83-97.

25. Stappenbelt B \& O’Neill L. 2007. Vortex-Induced Vibration of Cylindrical Structures with Low Mass Ratio, The Seventeenth International Offshore and Polar Engineering Conference. 2714-2721.

26. Sumer BM, Fredsoe J. 1997. Hydrodynamics around cylindrical structures. Singapore: World Scientific.

27. Thorsen MJ, Sævik S and Larsen CM. 2016. Time domain simulation of vortexinduced vibrations in stationary and oscillating flows. Journal of Fluids and Structures. (61): 1-19.

28. Triantafyllou, M, S, Hover, F, S, Yue, D, K, P (2003). "Vortex-induced vibrations of slender structures in shear flow,” Proceedings of the IUTAM Symposium on Coupled Fluid Structure Interaction Using Analysis, Computations and Experiments, Rutgers, NJ, USA, pp 1-6.

29. Violette R, Langre DE and Szydlowski J. 2007. Computation of vortex-induced vibrations of long structures using a wake oscillator model: Comparison with DNS and experiments. Computers and Structures. 85(11-14):1134-1141.

30. Xu J, Wang D, Huang H, Duan M, Gu J \& An C. 2017. A vortex-induced vibration model for the fatigue analysis of a marine drilling riser. Ships and Offshore Structures. 12(1): 280-287.

31. Xu K, Ge Y, \& Zhang D. 2015. Wake oscillator model for assessment of vortexinduced vibration of flexible structures under wind action. Journal of Wind Engineering and Industrial Aerodynamic. 136: 192-200. 